\title{
Deflection of a Web-Core Sandwich Beam in Weak Direction
}

\author{
Yang Jie*
}

School of Engineering, Design and Technology, University of Bradford, Richmond Road, Bradford, West Yorkshire, UK, $B D 71 D P, U K$

\begin{abstract}
The method for calculating the deflection of a web-core sandwich panel in its weak direction is based on complex shell and plate theory. A simple method was presented by assuming inflection points are located at the mid-span of the facing plate segment between two adjacent web cores. It also assumes that an inflection point also exists at the midheight of the web core. Based on these assumptions, a very simple equation was proposed. However, these assumptions are necessary to be evaluated for its suitability since the geometry of a web-core sandwich can be much different in theoretical design. This study then carries out a parametric study to find the validity range of the presented equation for calculating the deflection of a web-core sandwich panel in its weak direction. In the parametric study, finite element method is used to analyze such deflection. The parametric study shows that the accuracy of the presented equation is acceptable when the flexural stiffness per length of the web core is not less than that of the facing plates. This conclusion can be considered in practical design stage.
\end{abstract}

Keywords: Deflection, web-core sandwich panel, weak direction, flexural stiffness.

\section{INTRODUCTION}

Sandwich structure has been used widely in aerospace, offshore and ship engineering. It also has great potential in other engineering fields such as civil or mechanical engineering. Its popular application is due to its many advantages. Besides light weight and high flexural strength, this structure is especially advantageous in resisting dynamic or blast action because it can absorb amounts of energy before failure. Among different sandwich structures, steel-core sandwich structure is a principal type. This structure uses cold-formed or hot-rolled steel as webs. Top and bottom facing plates are made of steel plates. According to the types of cross section, webs can be designed with web-core, C-core, Z-core, I-core, V-core, box-core, truss-core and so on. Web-core is the simplest structural form for the web in a steel-core sandwich panel. A steel plate is welded onto the top and the bottom facing plates by using laser welding technique to form a web-core sandwich panel. The web-core is placed only in one direction, and this causes the web-core sandwich panel has different properties in two directions. Along the direction of the web-core placement, the shear stiffness is stronger, and this direction is called strong direction. Perpendicular to the strong direction, the shear stiffness is much weaker due to no placement of web-cores, and this direction is called weak direction.

In the literature, many research studies have been reported to investigate the behavior of steel-core sandwich

*Address correspondence to this author at the School of Engineering, Design and Technology, University of Bradford, Richmond Road, Bradford, West Yorkshire, UK, BD7 1DP; Tel: +44 7541775959;

E-mail: J.Yang14@student.bradford.ac.uk structures. The conducted studies provided theoretical analysis, finite element simulation and experimental investigation. A simple method to analyze the sandwich structures is to present theoretical or empirical equations for calculating elastic constants in two directions. Then, a sandwich panel can be simplified a simple plate with different elastic constants in two directions. It is clear that the accuracy of the proposed equations for calculating the elastic constants is critical in determining the reliability of the analysis for a sandwich panel. Chen et al., [1], Nordstrand [2], Fung et al., [3-5] gave some expressions of these elastic constants obtained from different methods. Regarding the web-core sandwich panel, Romanoff and Varsta [6-8] studied its bending behavior by using plate theory. They also verified the accuracy of the theoretical results calculated from their presented equations. Using plate theory, they analyzed the stress distribution in a web-core sandwich beam. Taczala and Banasiak [9] carried out a study on the buckling of web-core panels.

Because web-core sandwich panel is actually a composite structure, it is generally complicated to conduct theoretical analysis. It accurate solution depends on the solving technique of plate theory. Shao [10] presented a simplified equation for calculating the deflection of a web-core sandwich panel in its weak direction. In his method, the web-core sandwich panel is simplified into a simple 2-D problem. By using beam theory, a very concise equation is given based on some assumptions. This equation is especially convenient for design purposes for its easy understanding and concise expression. However, the author also thought this equation must be evaluated in further study because its accuracy depends much on some assumptions. Unfortunately, the validity range of the presented equation was not provided. This 


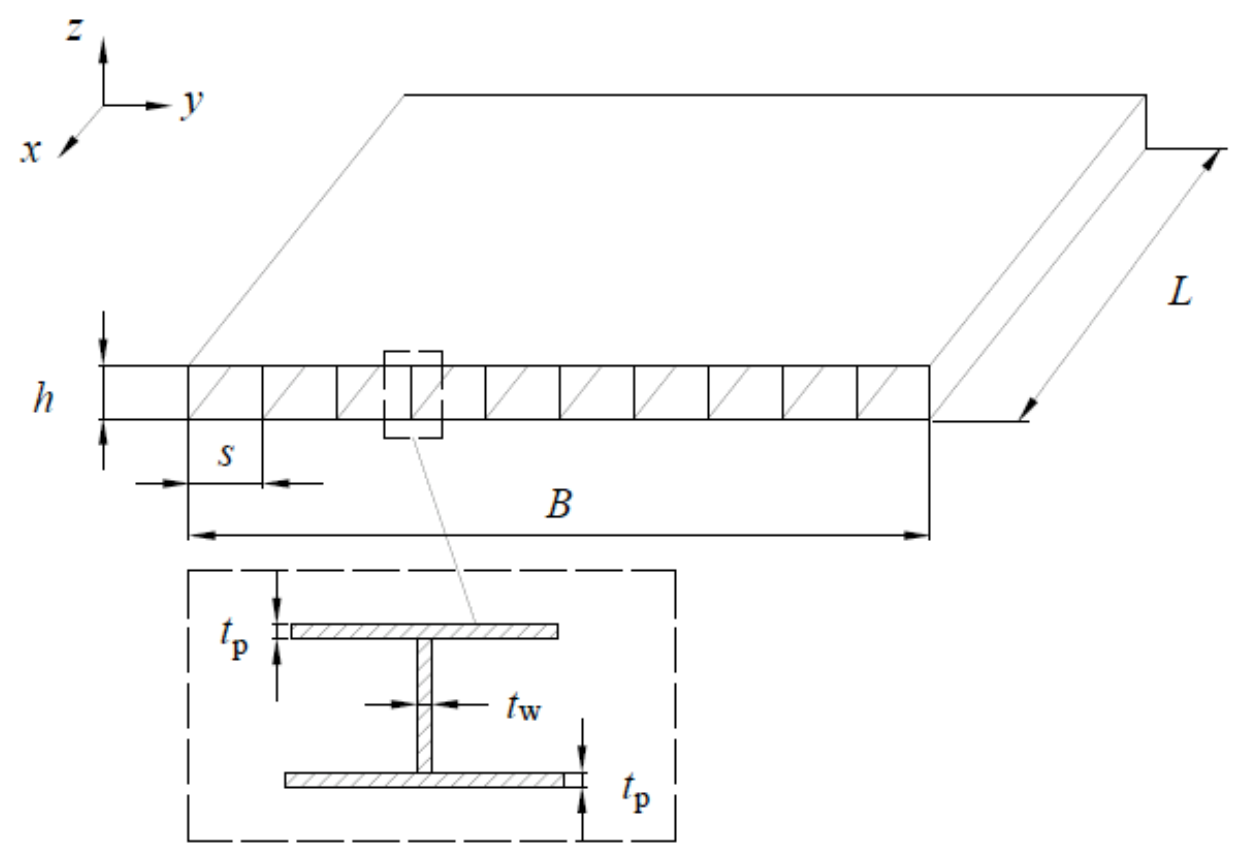

Fig. (1). A web-core sandwich panel.

paper then aims to conduct a further study to investigate the validity range of the equation proposed by Shao [10].

\section{REVIEW ON SHAO'S EQUATION (2012)}

\section{Basic Assumptions}

To provide a clear understanding, it is necessary to give a brief introduction on the simplified method presented by Shao [10]. Fig. (1) shows a web-core sandwich panel. $S$ and $h$ denote the distance between two adjacent webs and the height between top and bottom facing plates respectively. $t_{\mathrm{w}}$ and $t_{\mathrm{p}}$ are the thicknesses of the web and of the facing plates. It is assumed that the top and the bottom facing plates have same thickness. $B$ and $L$ are the panel length and panel width respectively. In the method presented by Shao [10], the following assumptions are specified: (1) all the webs have same dimensions in geometry, and any two adjacent webs are placed in a fixed distance; (2) the connection between the web and the facing plates are rigid; (3) a typical segment including two facing plates and a web as shown in Fig. (1) has identical deformation. This means same segment can be placed next to a typical segment, and both equilibrium and compatibility conditions will still be satisfied. This is called recurrence condition; (4) the total deformation of the segment includes shear deformation and flexural deformations. For the shear deformation, inflection point exists both at the facing plates and at the web core. The inflection points are located at the mid-points of the facing plates and the webs.

\section{Equations for Calculating the Deflection of a Web-Core Sandwich Panel in Weak Direction}

Based on the previous assumptions, Shao [10] presented an equation to calculate the shear deformation of a web-core sandwich panel in its weak direction. The equation is listed as follow:

$$
\Delta_{s}=n \frac{V s^{2}}{12}\left(\frac{1}{k_{w}}+\frac{1}{k_{f}}\right)
$$

where $n$ is number of typical segments; $V$ is shear force at two ends of a typical segment; $k_{\mathrm{w}}$ and $k_{\mathrm{f}}$ are bending stiffness per unit length for the web core and for the facing plates respectively.

In Eq. (1), $k_{\mathrm{w}}$ and $k_{\mathrm{f}}$ can be calculated from the following equation:

$$
\left\{\begin{array}{l}
k_{\mathrm{w}}=\frac{E_{\mathrm{w}} I_{\mathrm{w}}}{\left(1-v_{\mathrm{w}}^{2}\right) h} \\
k_{\mathrm{f}}=\frac{E_{f} I_{f}}{\frac{s}{2}\left(1-v^{2}\right)}
\end{array}\right.
$$

where $E_{\mathrm{w}}$ and $E_{\mathrm{f}}$ are the elastic modulus for the web core and for the facing plates respectively; $I_{\mathrm{w}}$ and $I_{\mathrm{f}}$ are the moment of inertia per unit width with respect to the mid-plane for the web core and for the facing plates respectively; $v$ is Poisson's ratio, and $I_{\mathrm{w}}=t_{\mathrm{w}}^{3} / 12, I_{\mathrm{f}}=t_{\mathrm{p}}^{3} / 12$.

Eq. (1) is used to calculate the shear deformation of a web-core sandwich panel in its weak direction. To calculate the flexural deformation $\Delta_{\mathrm{b}}$, beam theory can be easily used.

\section{PARAMETRIC STUDY}

\section{Parametric Models}

Eqs. (1) and (2) provide a simple method for calculating the deflection of a web-core sandwich panel in weak direction. However, this equation is only valid when the previous assumptions are satisfied. It is critical to present a validity 
Table 1. Web-Core Sandwich Panels

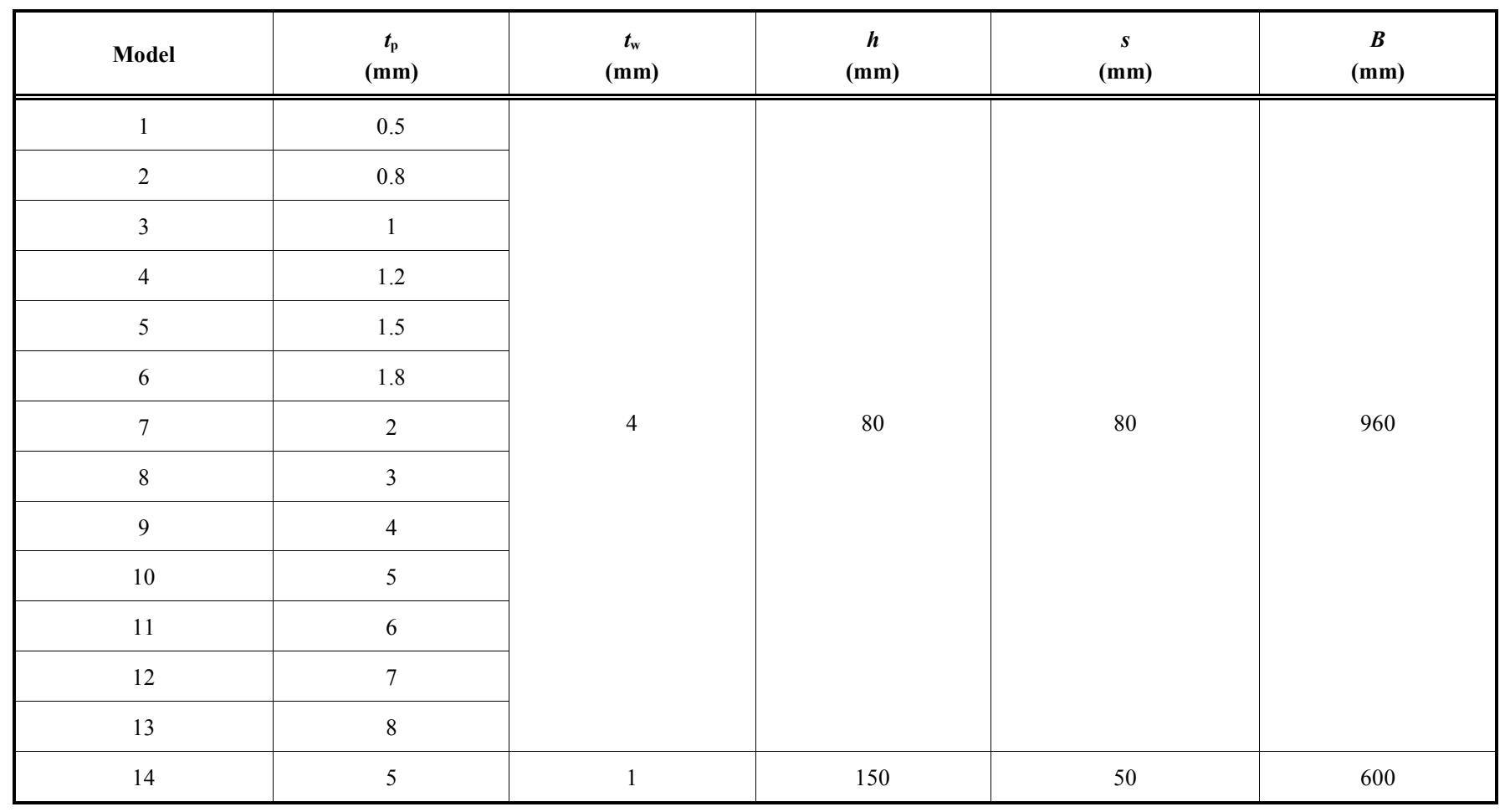

Table 2. Web-Core Sandwich Panels

\begin{tabular}{|c|c|c|c|c|c|}
\hline Model & $\begin{array}{c}t_{\mathrm{p}} \\
(\mathrm{mm})\end{array}$ & $\begin{array}{c}t_{\mathrm{w}} \\
(\mathrm{mm})\end{array}$ & $\begin{array}{c}h \\
(\mathrm{~mm})\end{array}$ & $\begin{array}{c}s \\
(\mathbf{m m})\end{array}$ & $\begin{array}{c}B \\
(\mathbf{m m})\end{array}$ \\
\hline 1 & \multirow{4}{*}{4} & \multirow{4}{*}{4} & 40 & \multirow{4}{*}{120} & \multirow{4}{*}{1440} \\
\hline 2 & & & 60 & & \\
\hline 3 & & & 120 & & \\
\hline 4 & & & 160 & & \\
\hline
\end{tabular}

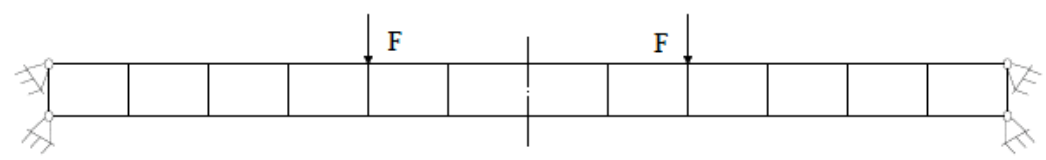

(a) Four point bending beams

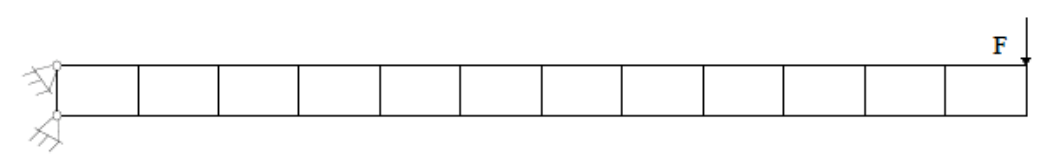

(b) Cantilever beams

Fig. (2). Analysis models in parametric study.

range in which Eq. (1) is accurate and reliable. This can be done through a parametric study on web-core sandwich panels with different geometries.

In the parametric study, overall models tabulated in Table $\mathbf{1}$ and in Table $\mathbf{2}$ are analyzed by using finite element method. In Table 1, the web-core sandwich models have different thickness ratios of the facing plate to the web core. In Table 2, the web-core models have different ratios of height to width of the segment.

The web-core sandwich panels in Table $\mathbf{1}$ are assumed to be four-point bending beams as illustrated in Fig. (2a). Accordingly, the web-core sandwich panels in Table $\mathbf{2}$ are as- 


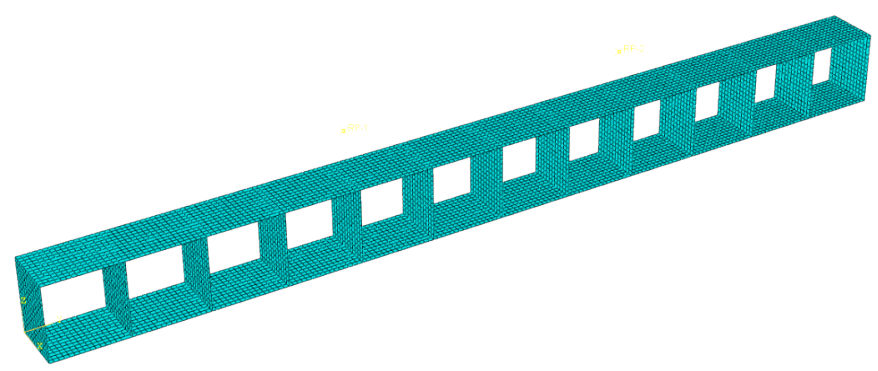

Fig. (3). Mesh of a web-core sandwich panel.

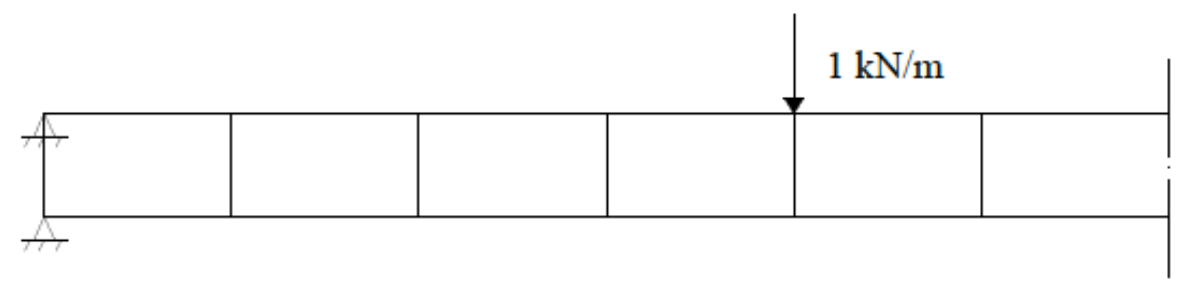

Fig. (4). Half model of a four point beam.

Table 3. Comparison between Predicted and Finite Element Results

\begin{tabular}{|c|c|c|c|c|c|c|}
\hline Model & $\begin{array}{c}w_{\mathrm{f}} \\
(\mathrm{mm})\end{array}$ & $\begin{array}{c}w_{\mathrm{s}} \\
(\mathrm{mm})\end{array}$ & $\begin{array}{c}w_{\mathrm{b}} \\
(\mathrm{mm})\end{array}$ & $\begin{array}{c}w_{\mathrm{e}}=w_{\mathrm{s}}+w_{\mathrm{b}} \\
(\mathrm{mm})\end{array}$ & $e$ & $\boldsymbol{k}_{\mathrm{w}} / \boldsymbol{k}_{\mathrm{f}}$ \\
\hline 1 & -36.9162 & -36.26 & -0.075 & -36.335 & $-1.57 \%$ & 256 \\
\hline 2 & -9.13162 & -8.976 & -0.047 & -9.023 & $-1.19 \%$ & 62.5 \\
\hline 3 & -4.7480 & -4.665 & -0.038 & -4.703 & $-0.95 \%$ & 32 \\
\hline 5 & -1.5070 & -1.48 & -0.025 & -1.505 & $-0.13 \%$ & 9.48 \\
\hline 6 & -0.9304 & -0.917 & -0.021 & -0.938 & $0.82 \%$ & 5.49 \\
\hline 7 & -0.7152 & -0.707 & -0.019 & -0.726 & $1.51 \%$ & 4 \\
\hline 8 & -0.3051 & -0.309 & -0.013 & -0.322 & $5.54 \%$ & 1.185 \\
\hline 11 & -0.1432 & -0.162 & -0.0063 & -0.168 & $17.7 \%$ & 0.148 \\
\hline 12 & -0.1310 & -0.155 & -0.0054 & -0.160 & $22.4 \%$ & 0.093 \\
\hline 13 & -0.1220 & -0.15 & -0.0047 & -0.155 & $26.8 \%$ & 0.0625 \\
\hline 14 & -1.274 & -6.64 & -0.0005 & -6.641 & $421.2 \%$ & 0.00133 \\
\hline
\end{tabular}

sumed to be cantilever beams as shown in Fig. (2b). A linear load with a magnitude of $1.0 \mathrm{~N} / \mathrm{mm}$ is applied at the $1 / 3 B$ and $2 / 3 B$ for the models in Table $\mathbf{1}$, and it is applied at the free end for the models in Table 2.

\section{Finite Element Analysis}

Finite element modeling for web-core sandwich panels can be implemented easily by using some popular commercial finite element software. ABAQUS, which is widely used in numerical simulation, is used in this study to conduct the analysis. Shell element is selected to mesh the overall struc- ture. The mesh of a web-core sandwich panel can be seen in Fig. (3).

For the models in Table 1, only half model can be analyzed due to the structural symmetry. The half model is shown in Fig. (4).

\section{RESULTS AND DISCUSSION}

For the models in Table $\mathbf{1}$, the predicted deflections and the finite element results are both tabulated in Table $\mathbf{3}$, where $w_{\mathrm{f}}$ is the finite element result; $w_{\mathrm{s}}$ and $w_{\mathrm{b}}$ are the predicted shear deformation and the flexural deformation respectively; 


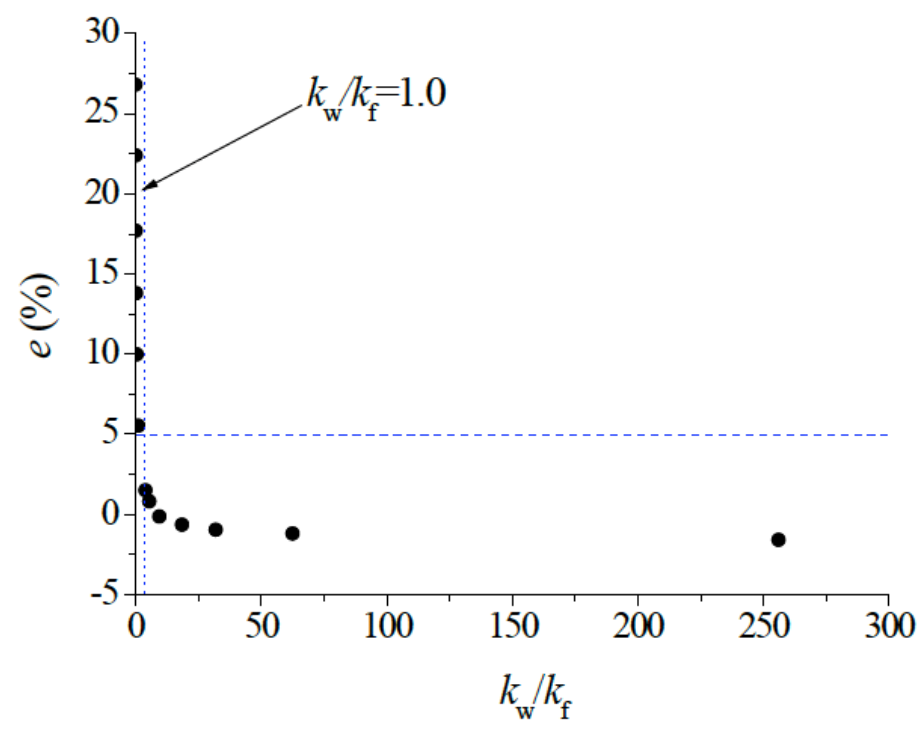

Fig. (5). Relationship between error and bending stiffness ratio.

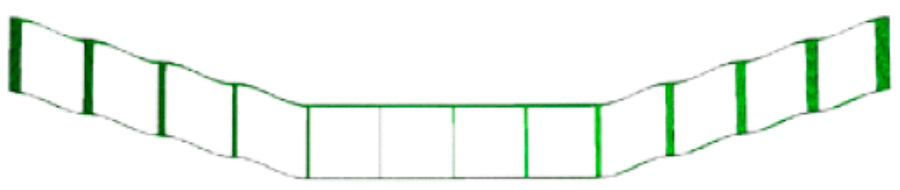

(a) $k_{\mathrm{w}} / k_{\mathrm{f}}=32.0$

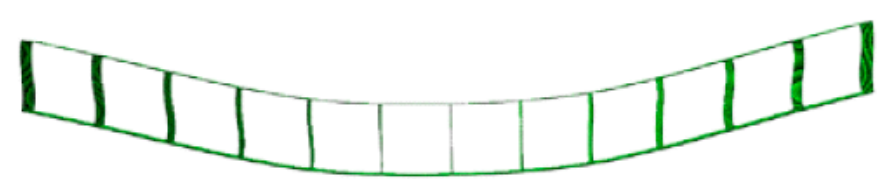

(b) $k_{\mathrm{w}} / k_{\mathrm{f}}=0.0625$

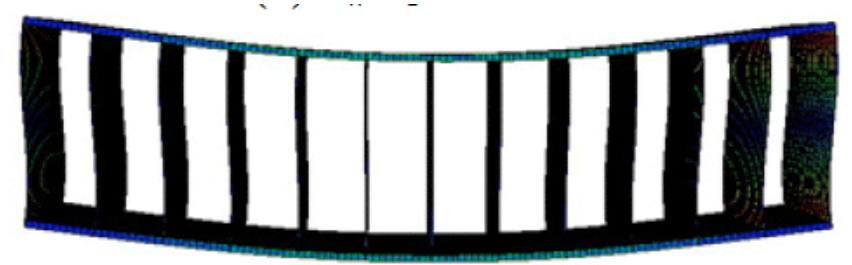

(c) $k_{\mathrm{w}} / k_{\mathrm{f}}=0.00133$

Fig. (6). Deformations of web-core sandwich panels with different flexural stiffness ratios.

$w_{\mathrm{e}}$ is the total deformation of the predicted result, and it is the sum of the shear deformation and the flexural deformation; $k_{\mathrm{w}} / k_{\mathrm{f}}$ is the bending stiffness per width ratio of the web to the facing plate; $e$ is the relative error between the finite element result and the predicted result, and it is defined as following equation:

$e=\frac{w_{\mathrm{e}}-w_{\mathrm{f}}}{w_{\mathrm{f}}} \times 100 \%$

In Table 3, $w_{\mathrm{s}}$ can be calculated from Eq. (1), while $w_{\mathrm{b}}$ can be easily calculated from simple beam theory as follow:

$w_{\mathrm{b}}=\frac{5\left(1-v^{2}\right) F B^{3}}{162 E I_{\mathrm{b}}}$

where $I_{\mathrm{b}}$ is the moment of inertia per unit width of the cross section about the neutral axis, $I_{\mathrm{b}}=t_{\mathrm{p}} h^{2} / 2$.
It can be seen from Table 3 that Eq. (1) can provide reasonably accurate estimation for the deflection of the fourpoint web-core sandwich beams when $k_{\mathrm{w}} / k_{\mathrm{f}}$ is bigger than 1.0. In this case, the relative error is not larger than $5.0 \%$, which is an acceptable prediction in engineering design. This means Shao's [10] equation is reliable when the bending stiffness per width unit of the web core is not less than that of the facing plate. The relationship between the relative error and the $k_{\mathrm{w}} / k_{\mathrm{f}}$ ratio is shown in Fig. (5), which also proves the above conclusion.

Fig. (6) shows the deformation of the sandwich models with different $k_{\mathrm{w}} / k_{\mathrm{f}}$ ratios. It can also be seen that when the $k_{\mathrm{w}} / k_{\mathrm{f}}$ ratio is bigger, each segment has much similar shear deformation. However, with the increase of the $k_{\mathrm{w}} / k_{\mathrm{f}}$ ratio, the deformation of each segment becomes much different. The assumption that the inflection point is located at the 
Table 4. Comparison between Predicted and Finite Element Results

\begin{tabular}{|c|c|c|c|c|c|c|}
\hline Model & $\begin{array}{c}w_{\mathrm{f}} \\
(\mathrm{mm})\end{array}$ & $\begin{array}{c}w_{\mathrm{s}} \\
(\mathrm{mm})\end{array}$ & $\begin{array}{c}w_{\mathrm{b}} \\
(\mathrm{mm})\end{array}$ & $\begin{array}{c}w_{\mathrm{e}}=w_{\mathrm{s}}+w_{\mathrm{b}} \\
(\mathrm{mm})\end{array}$ & $e$ & $\boldsymbol{k}_{\mathrm{w}} / \boldsymbol{k}_{\mathrm{f}}$ \\
\hline 1 & -2.6035 & -1.1927 & -1.3740 & -2.5667 & $-1.41 \%$ & 1.5 \\
\hline 2 & -2.0225 & -1.4313 & -0.6107 & -2.042 & $0.96 \%$ & 1.0 \\
\hline 3 & -2.1993 & -2.1469 & -0.1527 & -2.2996 & $4.56 \%$ & 0.5 \\
\hline 4 & -2.7587 & -2.6240 & -0.0859 & -2.7099 & $-1.77 \%$ & 0.375 \\
\hline
\end{tabular}

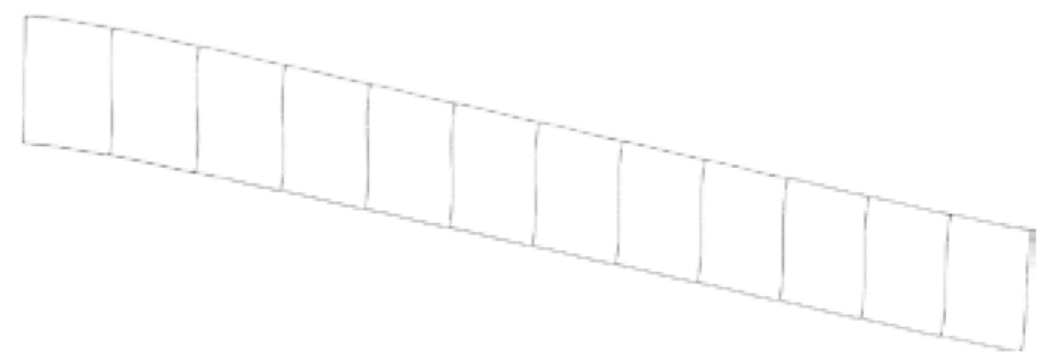

Fig. (7). Deformation of the cantilever web-core sandwich beam.

mid-span of the facing plate and the web core is not satisfied in this case.

Table 4 shows the effect of $h$ and $s$ on the accuracy of the presented equation. The deflection caused by flexural action is calculated from the following equation which can be obtained easily from beam theory:

$w_{\mathrm{b}}=\frac{\left(1-v^{2}\right) F B^{3}}{3 E I_{\mathrm{b}}}$

The finite element results agree quite well with predicted values since all the relative errors are within a range of less than $5 \%$. It seems that parameters $h$ and $s$ have minor effect on the accuracy of the presented equation. The typical deformation of Model 4 in Table 4 is shown in Fig. (7). It is clear that each segment has similar deformation in the cantilever beam.

\section{SUMMARY}

This paper conducted a parametric study to evaluate the accuracy and reliability of a simple equation presented by Shao [10] for predicting the deflection of a web-core sandwich panel in weak direction. From parametric study, it has been found that the equation can provide reliably and reasonably accurate estimation when the flexural stiffness per unit width of the web core is not less than that of the facing plate. In this case, the assumptions given by Shao [10] can be satisfied.

\section{CONFLICT OF INTEREST}

The authors confirm that this article content has no conflicts of interest.

\section{ACKNOWLEDGEMENT}

Declared none.

\section{REFERENCES}

[1] Q.H. Cheng, H.P. Lee and C. Lu, "A numerical analysis approach for evaluating elastic constants of sandwich structures with various cores", Compos. Struct., vol. 74, pp. 226-236, 2006.

[2] T. Nordstrand, "Transverse shear stiffness of structural core sandwich”, Compos. Struct., vol. 27, pp. 317-329, 1994.

[3] T.C. Fung, K.H. Tan and T.S. Lok, "Shear stiffness $D_{\mathrm{Qy}}$ for C-core sandwich panels", J. Struct. Eng. ASCE, vol. 122, pp. 958-966, 1996.

[4] T.C. Fung and K.H. Tan, "Shear stiffness for Z-core sandwich panels", J. Struct. Eng. ASCE, vol. 124, pp. 809-816, 1998.

[5] T.C. Fung, K.H. Tan and T.S Lok, "Elastic constants for Z-core sandwich panels", J. Struct. Eng. ASCE, vol. 120, pp. 3046-3065, 1994.

[6] J. Romanoff and P. Varsta, "Bending response of web-core sandwich beams", Compos. Struct., vol. 73, pp. 478-487, 2006.

[7] J. Romanoff and P. Varsta, "Bending response of web-core sandwich plates”, Compos. Struct., vol. 81, pp. 292-302, 2007.

[8] J. Romanoff, P. Varsta and A. Klanac, "Stress analysis of homogenized web-core sandwich beams", Compos. Struct., vol. 79, pp. 411-422, 2007.

[9] M. Taczala and W. Banasiak, "Buckling of I-core sandwich panels", J. Theor. Appl. Mech., vol. 42, pp. 335-348, 2004.

[10] Y.B. Shao, "A simplified prediction on deflection of web-core sandwich beam in weak direction", Appl. Mech. Mater., vol. 166169 , pp. 226-229, 2012.

(C) Yang Jie; Licensee Bentham Open.

This is an open access article licensed under the terms of the Creative Commons Attribution Non-Commercial License (http://creativecommons.org/licenses/by-nc/3.0/) which permits unrestricted, non-commercial use, distribution and reproduction in any medium, provided the work is properly cited. 